\title{
TỐI ƯU HÓA NĂNG LƯợNG TIÊU THỤ MẠNG CẢM BIẾN KHÔNG DÂY
}

\section{Lại Thị Nhung ${ }^{a}$, Nguyễn Thị Hòaa , Lê Đăng Nguyên', Nguyễn Gia Như ${ }^{c}$, Đặng Thanh Hải ${ }^{d^{*}}$}

${ }^{a}$ Khoa Khoa học Co bản, Truò̀ng Đại học Điều duõng Nam Định, Nam Định, Việt Nam

${ }^{b}$ Khoa Công nghệ Thông tin, Truờng Đại học Hải Phòng, Hải Phòng, Việt Nam

${ }^{c}$ Khoa Công nghệ Thông tin, Truờng Đại học Duy Tân, Đà Nẵng, Việt Nam

${ }^{d}$ Khoa Công nghệ Thông tin, Truờng Đại học Đà Lạt, Lâm Đồng, Việt Nam

"Tác giả liên hệ: Email: haidt@dlu.edu.vn

\section{Lịch sử bài báo}

Nhận ngày 02 tháng 03 năm 2018

Chỉnh sửa ngày 29 tháng 03 năm 2018 | Chấp nhận đăng ngày 14 tháng 04 năm 2018

\section{Tóm tắt}

Trong mạng cảm biến không dây, các cảm biến được triển khai nhằm mục đích thu thập thông tin tù khu vực muc tiêu. Các thông tin thu thập của các cảm biến sẽ được chuyển đến trạm cơ sở bằng sóng vô tuyến. Tù đây, dũ liẹu được phân tích, xủ lý để đưa ra các quyết định. Một trong các vấn đề của mạng cảm biến không dây là cần phải tiết kiệm năng luợng tiêu thu, kéo dài tuổi tho của mạng. Trong bài báo này, chúng tôi đề xuất giải pháp triển khai mạng cảm biến bằng cách phân cụm và kết hợp chọn nút chủ cưm để tối uu hóa năng luợng tiêu thu và kéo dài tuổi thọ của mang. Việc phân tích so sánh thuật toán đề xuất với thuật toán phân cưm truyền thống LEACH, LEACH-C, K-Mean và FCM sẽ khẳng định tính hiệu quả của giải pháp đã đề xuất.

Từ khóa: Mạng cảm biến không dây; Năng lượng; Nút chủ; Phân cụm.

Mã số định danh bài báo: http://tckh.dlu.edu.vn/index.php/tckhdhdl/article/view/430

Loại bài báo: Bài báo nghiên cứu gốc có bình duyệt

Bản quyền @ 2018 (Các) Tác giả.

Cấp phép: Bài báo này được cấp phép theo CC BY-NC-ND 4.0 


\title{
OPTIMIZING ENERGY CONSUMPTION FOR WIRELESS SENSOR NETWORKS
}

\section{Lai Thi Nhunga, Nguyen Thi Hoa ${ }^{a}$, Le Dang Nguyen ${ }^{b}$, Nguyen Gia Nhuc, Dang Thanh Hai ${ }^{\text {** }}$}

\footnotetext{
${ }^{a}$ The Faculty of Basic Science, Namdinh University of Nursing, Namdinh, Vietnam ${ }^{b}$ The Faculty of Information Technology, Haiphong University, Haiphong, Vietnam ${ }^{c}$ The Faculty of Information Technology, Duy Tan University, Danang, Vietnam ${ }^{d}$ The Faculty of Information Technology, Dalat University, Lamdong, Vietnam

*Corresponding author: Email: haidtt@dlu.edu.vn
}

\section{Article history}

Received: March 02 ${ }^{\text {nd }}, 2018$

Received in revised form: March $29^{\text {th }}, 2018$ | Accepted: April $14^{\text {th }}, 2018$

\begin{abstract}
In wireless sensor networks, sensors are deployed and aim to gather information from the target area. The information collected by the sensors will be transmitted to the base station by radio waves. From here, the data is analyzed and processed to make decisions. One of the problems of the wireless sensor network is the need to save energy consumption, extending the life of the network. In this paper, we propose a solution for deploying sensor networks by clustering and combining cluster head selection to optimize power consumption and extend the life of the network. Comparative analysis of algorithms proposed with traditional clustering algorithms LEACH, LEACH-C, K-Mean, and FCM will confirm the effectiveness of the proposed solution.
\end{abstract}

Keywords: Cluster head; Clustering; Energy consumption; Wireless sensor network.

Article identifier: http://tckh.dlu.edu.vn/index.php/tckhdhdl/article/view/430

Article type: (peer-reviewed) Full-length research article

Copyright @ 2018 The author(s).

Licensing: This article is licensed under a CC BY-NC-ND 4.0 


\section{GIỚI THIỆU}

Một mạng cảm biến không dây WSN (Wireless Sensor Network) bao gồm một tập các cảm biến được triển khai trong một khu vực mục tiêu nhằm mục đích thu thập các thông tin từ môi trường. Các thông tin này được chuyển đến nút trung tâm gọi là $B S$ (Base Station) bằng sóng vô tuyến. Từ đó dữ liệu được phân tích, xử lý bởi người sử dụng để đưa ra các quyết định cho hệ thống (Akbarzadeh, Gagné, Parizeau, Argany, \& Mostafavi, 2013).

Mạng cảm biến không dây được ứng dụng nhiều trong thực tế như giám sát môi trường, phát hiện xâm nhập, cảnh báo cháy rừng, thảm họa môi trường, giao thông, theo dõi sức khỏe con người (Huang \& Tseng, 2005). Một vấn đề quan trọng của mạng cảm biến là cần phải xây dựng cấu trúc liên kết các cảm biến nhằm tối ưu hóa năng lượng tiêu thụ của các cảm biến, kéo dài tuổi thọ của mạng. Các cảm biến trong mạng thường được liên kết thành một trong các cấu trúc liên kết như: Cấu trúc hình sao; Cấu trúc phân cấp; và Cấu trúc tập trung (Yick, Mukherjee, \& Ghosal, 2008).

- Cấu trúc hình sao: Trong cấu trúc hình sao thì mỗi cảm biến được kết nối trực tiếp đến $B S$. Tất cả cảm biến trong mạng đều có vai trò và chức năng như nhau. Các cảm biến cộng tác với nhau để thực hiện nhiệm vụ thu thập thông tin từ môi trường và chuyển đến $B S$ (Hoang, Kumar, \& Panda, 2010). Tuy nhiên, với cấu trúc này, các cảm biến cộng tác thu thập thông tin dư thừa từ môi trường và tiêu thụ năng lượng nhiều hơn do khoảng cách trực tiếp đến $B S$ xa.

- Cấu trúc phân cấp: Trong cấu trúc phân cấp, mỗi nút cảm biến kết nối trực tiếp đến một nút ở cấp cao hơn trong cây và sau đó đến $B S$, dữ liệu được định tuyến từ nút thấp nhất trong mạng đến $B S$. Ý tưởng của phương pháp này khá đơn giản, vì phạm vi sóng vô tuyến của các nút cảm biến bị giới hạn, do đó cách tốt nhất là nó truyền dữ liệu đến nút lân cận với nó trong cây và sau đó đến $B S$. Trong cấu trúc này, các nút cảm biến ở cấp cao tiêu thụ nhiều năng lượng hơn các nút cảm biến ở cấp dưới do đó các nút cảm biến ở cấp cao càng gần với $B S$ sẽ nhanh chóng cạn kiệt năng lượng làm giảm hiệu suất của mạng.

- Cấu trúc tập trung: Trong cấu trúc này các cảm biến được tổ chức thành từng nhóm, trong đó một nút được chọn làm nút chủ (Cluster Head - $\mathrm{CH}$ ) các nút còn lại là non- $\mathrm{CH}$. Các non- $\mathrm{CH}$ có nhiệm vụ thu thập các thông tin từ môi trường và chỉ chuyển thông tin đến $\mathrm{CH}$ khi cần thiết. Trong khi đó các $C H$ có nhiệm vụ thu thập, phân tích loại bỏ thông tin dư thừa từ các $n o n-C H$ và chuyển đến $B S$ (Hoang $\&$ ctg., 2010).

Như vậy, việc xây dựng cấu trúc liên kết mạng cảm biến theo kiểu tập trung hay phân cụm cảm biến sẽ tiết kiệm năng lượng tiêu thụ cho toàn mạng. Tuy nhiên, để các 
cụm cảm biến tiêu tốn năng lượng ít nhất hay kéo dài tuổi thọ của mạng, thì các nút cảm biến trong cụm phải được tối ưu năng lượng tiêu thụ cho việc cảm nhận thông tin, truyền dữ liệu đến nút $C H$. Trong bài báo này, chúng tôi áp dụng thuật toán phân cụm mờ kết hợp lập lịch hoạt động của các cảm biến gần nhau trong cụm để tối ưu năng lượng tiêu thụ và giảm thông tin dư thừa.

Phần còn lại của bài viết được tổ chức như sau. Mục 2 trình bày một số thuật toán phân cụm cho mạng cảm biến không dây. Mục 3 trình bày mô hình tiêu thụ năng lượng và tối ưu hóa tổng năng lượng tiêu thụ của toàn mạng dựa trên thuật toán phân cụm mờ kết hợp lập lịch hoạt động của các cảm biến. Các kết quả thực nghiệm sẽ được trình bày trong Mục 4. Cuối cùng, kết luận được đưa ra trong Mục 5.

\section{MỘT SỐ PHƯƠnG PHÁP PHÂN CỤM}

\subsection{Low energy adaptive clustering hierarchy (LEACH)}

LEACH (Yick \& ctg., 2008) là phân cụm phân cấp thích ứng năng lượng thấp. Mục đích chính của LEACH là kéo dài thời gian sống của mạng, giảm năng lượng tiêu thụ của mỗi nút, sử dụng dữ liệu tập trung để giảm thông điệp truyền dữ liệu trong mạng. Một đặc điểm của $\mathrm{LEACH}$ là tự tổ chức các cảm biến thành cụm mà trong đó có một nút được chọn làm nút chủ $\mathrm{CH}$. Các nút thành viên là non- $\mathrm{CH}$ có nhiệm vụ thu thập thông tin từ môi trường rồi chuyển đến nút $\mathrm{CH}$. Nút $\mathrm{CH}$ nhận dữ liệu từ các nút non- $\mathrm{CH}$ trong cụm, thực hiện phân tích, xử lý loại bỏ thông tin dư thừa và chuyển tiếp đến $B S$. Do đó các nút $\mathrm{CH}$ sẽ tiêu thụ năng lượng nhiều hơn các nút non- $\mathrm{CH}$. LEACH dề xuất xoay vòng ngẫu nhiên vai trò nút $C H$ cho các nút non- $C H$ trong cụm để tránh việc tiêu thụ năng lượng chỉ tập trung vào một nút.

Khi bắt đầu một vòng mới trong $\mathrm{LEACH}$, nó sẽ quyết định có hay không một nút cảm biến có thể trở thành nút $C H$ dựa trên xác suất $P$ trở thành $C H$ trong mạng và số lần nút đó trở thành nút $C H$ trước đó. Mỗi nút chọn một số ngẫu nhiên $S$ trong đoạn [0, 1]. Nếu giá trị này nhỏ hơn ngưỡng $T(n)$ thì nút đó trở thành $C H$ của vòng hiện thời. Giá trị ngưỡng này được xác định bởi công thức (1) như sau:

$$
T(n)=\left\{\begin{array}{lc}
0 & n \in G \\
\frac{P}{1-P \times(r \bmod (1 / P))} & n \notin G
\end{array}\right.
$$

Trong đó: $r$ là vòng hiện tại; $G$ là tập hợp các nút đã trở thành $C H$ trong $1 / P$ vòng trước.

Khoảng cách giữa các cảm biến trong môi trường 3D được tính như công thức (2) trong đó $\left(x_{s_{i}}, y_{s_{i}}, h_{s_{i}}\right)$ là tọa độ của cảm biến $s_{i}$ trong địa hình. 


$$
d\left(s_{i}, s_{j}\right)=\sqrt{\left(x_{s_{i}}-x_{s_{j}}\right)^{2}+\left(y_{s_{i}}-y_{s_{j}}\right)^{2}+\left(h_{s_{i}}-h_{s_{j}}\right)^{2}}
$$

\subsection{LEACH-C}

LEACH-C tương tự như LEACH nhưng nó sẽ quyết định nút trở thành $C H$ là nút có năng lượng còn lại lớn và vị trí của nó trong địa hình. Mặt khác, LEACH chọn nút $C H$ không dựa vào năng lượng còn lại mà dựa vào số lần trở thành $C H$ trong các vòng trước đó. Điều này làm cho các cảm biến ở xa $B S$ sẽ nhanh chóng cạn kiệt năng lượng. Hơn nữa, việc chọn $C H$ không dựa vào sự phân bố của các cảm biến sẽ hình thành các cụm chồng chéo. Để khắc phục các nhược điểm này, $\mathrm{LEACH}-\mathrm{C}$ cho phép các cảm biến gửi thông tin về năng lượng còn lại và vị trí đến $B S$ (Yin, Shi, Li, \& Zhang, 2006).

Kaur và Gupta (2015) mô tả LEACH-C sử dụng thuật toán PBO (Pollination Based Optimization) để chọn các $C H$ dựa vào năng lượng còn lại và khoảng cách. Nếu có hai nút cảm biến có cùng năng lượng còn lại thì LEACH-C chọn cảm biến làm $C H$ dựa vào khoảng cách từ nó đến $B S$. LEACH-C đã cải tiến hiệu quả $\mathrm{LEACH}$ về năng lượng, chi phí và kéo dài tuổi thọ của mạng.

\subsection{K-Means}

Một phương pháp phân cụm khác dựa trên năng lượng còn lại của các cảm biến là thuật toán K-Means. Không giống như $\mathrm{LEACH}$ và $\mathrm{LEACH}-\mathrm{C}$, thuật toán $\mathrm{K}-\mathrm{Means}$ tìm kiếm cảm biến gần với tâm cụm chọn làm $C H$. Tham số quan trọng nhất thuật toán K-Means chính là khoảng cách Euclidean. Thuật toán K-Means lặp đi lặp lại việc gom cụm các cảm biến có khoảng cách ngắn nhất với tâm cụm, sau mỗi lần lặp, tâm cụm được tính lại, thuật toán dừng khi tâm cụm không thay đổi. Điều này đảm bảo năng lượng tiêu thụ của các cảm biển trong cụm cho việc truyền dữ liệu đến $C H$ của chúng là ít nhất. Tuy nhiên, cũng giống như các phương pháp phân cụm khác, các nút $C H$ luôn tiêu tốn năng lượng nhiều hơn các cảm biến khác vì chúng có nhiệm vụ nhận dữ liệu từ các cảm biến thành viên trong cụm, rồi tổng hợp loại bỏ dữ liệu dư thừa sau đó mới chuyển tiếp đến $B S$. Để đảm bảo cân bằng năng lượng giữa các cảm biến, kéo dài tuổi thọ của mạng, thuật toán K-Means thực hiện phân cụm xoay vòng với số cụm được khởi tạo mỗi lần khác nhau.

\subsection{Fuzzy C-Means (FCM)}

FCM là một trong các phương pháp được áp dụng rộng rãi trong logic mờ được đưa ra bởi Bezdek, Ehrlich, và Full (1984). Các cảm biến được gom thành cụm với độ thuộc của FCM giúp tối ưu hóa các cụm dựa vào việc giảm thiểu khoảng cách giữa các cảm biến và tâm cụm. Phương pháp này đảm bảo các cụm hình thành một cách thống nhất trong WSN mà các cảm biến được triển khai ngẫu nhiên. Các cụm được hình thành với các cảm biến có mật độ cao. Do đó, việc truyền dữ liệu được cân bằng giữa các $C H$ 
và năng lượng tiêu thụ cũng được cân bằng giữa các cảm biến trong nội cụm. Phương pháp FCM được giải quyết nhờ vào công thức (3) và công thức (4). Trong đó, $u_{i j}$ là độ thuộc của cảm biến $j$ vào cụm $i, d_{i j}$ là khoảng cách giữa cảm biến $j$ và tâm cụm $i$.

$$
\begin{gathered}
J=\sum_{i=1}^{c} \sum_{j=1}^{N} u_{i j}^{m} d_{i j}^{2} \\
\left\{\begin{array}{c}
u_{i j} \in[0,1] \\
\sum_{j=1}^{c} u_{i j}=1 \\
k=1, . ., N ; j=1, . ., c
\end{array}\right.
\end{gathered}
$$

Độ thuộc của các thành viên cảm biến và các tâm cụm được tính như các công thức (5) và (6).

$$
\begin{aligned}
& u_{i j}=\frac{1}{\sum_{k=1}^{c}\left(\frac{d_{i j}}{d_{k j}}\right)^{\frac{2}{m-1}}} \\
& V_{j}=\frac{\sum_{k=1}^{N} u_{k j}^{m} X_{k}}{\sum_{k=1}^{N} u_{k j}^{m}}
\end{aligned}
$$

Sau khi các cụm được hình thành, $B S$ chọn nút cảm biến gần nhất với tâm cụm làm $C H$ và việc tái phân cụmm được thực hiện sau một chu kỳ thời gian để đảm bảo năng lượng tiêu thụ được cân bằng giữa các $C H$ và non- $C H$ trong cụm.

\section{MÔ HÌNH ĐỀ XUẤT}

Giả sử có một địa hình $3 \mathrm{D}$ và một mạng cảm biến bao gồm $N$ cảm biến. Địa hình 3D được định nghĩa theo chuẩn DEM (Digital Elevation Model) (Nguyen, Dang, Le, \& Le, 2015) và được biểu diễn bằng một ma trận theo hàng, cột và giá trị ma trận chính là độ cao của tọa độ tương ứng theo hàng, cột. Triển khai một mạng cảm biến không dây đòi hỏi các cảm biến và $B S$ phải có một vị trí cố định trên địa hình. Mô hình mạng xem xét có các cảm biến đồng nhất về cấu trúc và tất cả cảm biến có một mức năng lượng khởi đầu như nhau. Mỗi nút cảm biến có trách nhiệm thu thập các thông số từ môi trường và định kỳ gởi thông tin đến nút nhận. Nút nhận là $C H$ hoặc $B S$. Nếu nút nhận là $C H$ thì nó có trách nhiệm tổng hợp và chuyển tiếp đển $B S$. Kênh vô tuyến dùng để chuyển dữ liệu có phạm vi là một hình cầu với bán kính $T_{r}$. Thông thường bán kính này ít nhất gấp đôi bán kính phủ sóng của cảm biến. Mặc khác, năng lượng tiêu thụ trong quá trình truyền dữ liệu từ nút $X$ đến nút $Y$ và ngược lại coi như giống nhau. Từ những giả định trên chúng tôi đề xuất một mô hình phân cụm mờ mới kết hợp việc chọn 
nút $C H$ dựa mô hình năng lượng tiêu thụ của các cảm biến trong mạng (FCM-CHS). Trong cấu trúc của một cảm biến không dây thì năng lượng được tiêu thụ vào các thành phần: Cảm nhận các thông số từ môi trường; Tổng hợp dữ liệu; Truyền nhận; và Khuếch đại dữ liệu. Chúng tôi xem xét mô hình tổn thất năng lượng cho quá trình truyền dữ liệu khoảng cách xa (năng lượng tổn thất $d^{4}$ ) và khoảng cách gần (năng lượng tổn thất $d^{2}$ ). Năng lượng tiêu thụ cho một nút $C H$ nhận, tổng hợp dữ liệu và chuyển tiếp đến $B S$ được tính như công thức (7).

$$
E_{C H}=l E_{\text {elec }}+l E_{D A}+l \varepsilon_{m p} d_{t o B S}^{4}
$$

Trong đó: $l$ là số bit của mỗi gói dữ liệu; $E_{D A}$ là năng lượng tiêu thụ cho việc tổng hợp dữ liệu và được đề nghị là $5 \mathrm{pJ} / \mathrm{bit} ; d_{t o B S}$ là khoảng cách từ nút $C H$ đến $B S$ trong môi trường $3 \mathrm{D}$ được tính bằng công thức $(2) ; E_{\text {elec là năng lượng tiêu thụ cho việc }}$ truyền, nhận mỗi bit dữ liệu và được đề nghị là $50 \mathrm{~nJ} /$ bit; và $\varepsilon_{f s}$, $\varepsilon_{m p}$ là chi phí năng lượng cho việc truyền một bit dữ liệu với một tỉ lệ bit lỗi chấp nhận được và được đề nghị là $10 \mathrm{pJ} / \mathrm{bit} / \mathrm{m} 2$ và $0.0013 \mathrm{pJ} / \mathrm{bit} / \mathrm{m} 4$.

Như vậy, năng lượng tiêu thụ của các nút non- $C H$ được tính như công thức (8).

$$
E_{\text {non-CH }}=l E_{\text {elec }}+l \varepsilon_{f_{s}} d_{t o C H}^{2}
$$

Trong đó: $d_{t o C H}$ là khoảng cách của nút cảm biến trong cụm đến $C H$ và tổng năng lượng tiêu thụ của mạng cho mỗi vòng được tính như công thức (9).

$$
E_{\text {Total }}=C \times E_{C H}+(N-C) \times E_{\text {non-CH }}
$$

Kết hợp các công thức (7), (8), và (9) chúng ta được công thức (10) để tính tổng năng lượng tiêu thụ như sau:

$$
E_{\text {Total }}=l\left(2 N E_{\text {elec }}+C E_{D A}+C \varepsilon_{m p} d_{\text {toBS }}^{4}+(N-C) \varepsilon_{f S} d_{t o H H}^{2}\right)
$$

Giả sử chọn $C$ cảm biến từ $N$ ban đầu làm $C H$, chúng ta phân bố các cảm biến còn lại vào $C$ cụm với mục tiêu tối thiểu năng lượng tiêu thụ như công thức (11).

$$
J=C \varepsilon_{m p} d_{t o B S}^{4}+(N-C) \varepsilon_{f s} d_{t o C H}^{2} \rightarrow \min
$$

Áp dụng logic mờ cho hàm năng lượng tại công thức (11) chúng ta được một hàm mục tiêu mới như công thức (12).

$$
J=C \varepsilon_{m p} \sum_{i=1}^{N} \sum_{j=1}^{c} u_{i j}^{m} \mid V_{j}-X_{B S}\left\|^{4}+(N-C) \varepsilon_{f s} \sum_{i=1}^{N} \sum_{j=1}^{c} u_{i j}^{m}\right\| X_{i}-V_{j} \|^{2} \rightarrow \min
$$


Trong đó $X_{B S}$ là tọa độ của $B S$. Với việc giới hạn bán truyền thông của các cảm biến, chúng tôi tích hợp các ràng buộc như công thức (13), (14), và (15).

$$
\begin{aligned}
& \sum_{i=1}^{c} u_{i j}=1 ; \quad u_{i j} \in[0,1] \quad \forall j=\overline{1, N} \\
& \left\|X_{i}-V_{j}\right\| \leq 2 T_{r} \\
& \left\|V_{j}-X_{B S}\right\| \leq 9 T_{r}
\end{aligned}
$$

Trong đó chúng tôi giả sử bán kính truyền thông của $B S$ gấp tám lần bán kính truyền thông của các cảm biến. Do đó, một cảm biến kết nối đến được $C H$ của nó thì khoảng cách của nó phải nhỏ hơn hoặc bằng $2 T_{r}$, và $C H$ kết nối được đến $B S$ nếu như khoảng cách giữa chúng nhỏ hơn hoặc bằng $9 T_{r}$. Bằng cách sử dụng phương pháp Lagrangian, độ thuộc và tâm cụm được tính theo các công thức (16), (17), và (18). Trong đó: $A, B$ là các hệ số; $a_{0}, d_{0}, s, \rho$, và $k$ là các tham số kiểm soát tốc độ hình dáng của hàm.

$$
\begin{aligned}
& u_{i j}=\left(\frac{\sum_{k=1}^{c} C m \varepsilon_{m p}\left\|V_{k}-X_{B S}\right\|^{4}+(N-C) \varepsilon_{f s}\left\|X_{i}-V_{k}\right\|^{2}}{C m \varepsilon_{m p}\left\|V_{j}-X_{B S}\right\|^{4}+(N-C) \varepsilon_{f i s}\left\|X_{i}-V_{j}\right\|^{2}}\right)^{\frac{1}{m-1}} \\
& V_{j}=V_{j}-\left(A \nabla_{V_{j}} J_{m}^{*}\left(V_{j}\right)-B\left(V_{j}-T\right)\right) \\
& T=\rho_{j} \sin \left[2 \pi\left(\frac{1}{s}\right)^{11-a_{0}-\frac{\left(1-a_{0}-d_{0}\right)(j-1)}{k}}\right], \quad j=1,2, \ldots, C
\end{aligned}
$$

Với $a_{0}, d_{0}$ nằm trong đoạn $[0,1], a_{0}+d_{0}<1 ; s>0 ; k \leq C$; và giá trị $J_{m}^{*}$ được tính như công thức (19).

$$
J_{m}^{*}=J_{m}+\lambda_{j}\left(\sum_{j=1}^{c} u_{i j}-1\right)+\frac{1}{2} \sum_{i=1}^{N} \sum_{j=1}^{c}\left(\max ^{2}\left(0, \mu_{i j}+g_{i j}\right)-\mu_{i j}^{2}\right)+\sum_{j=1}^{c}\left(\max ^{2}\left(0, \xi_{j}+h_{j}\right)-\xi_{j}^{2}\right)
$$

Trong đó:

$$
\begin{aligned}
& g_{i j}=g\left(V_{j}, X_{i}\right)=\left\|X_{i}-V_{j}\right\|-2 T_{r} \\
& h_{j}=h\left(V_{j}\right)=\left\|V_{j}-X_{B S}\right\|-9 T_{r}
\end{aligned}
$$

Giá trị $\mu_{\mathrm{ij}}, \xi_{\mathrm{j}}$ được tính như sau: 


$$
\begin{aligned}
& \frac{\partial J_{m}^{*}}{\partial \mu_{i j}}=\max \left(0, \mu_{i j}+g_{i j}\right)-\mu_{i j} \\
& \frac{\partial J_{m}^{*}}{\partial \xi_{j}}=\max \left(0, \xi_{j}+h_{j}\right)-\xi_{j}
\end{aligned}
$$

Như vậy, sau phân cụm mờ thì các cụm cảm biến hình thành tối ưu năng lượng tiêu thụ cho quá trình chuyển dữ liệu từ các nút $n o n-C H$ đến nút $C H$, từ các nút $C H$ đến $B S$. Tuy nhiên, việc chọn $C H$ xoay vòng cho các cụm sao cho tối ưu năng lượng tiêu thụ và kéo dài tuổi thọ của mạng tức là năng lượng còn lại của nút $C H$ lớn và khoảng cách từ $C H$ dến các nút thành viên trong cụm ngắn nhất. Hàm mục tiêu cho việc tối thiểu năng lượng tiêu thụ cho quá trình truyền dữ liệu từ non- $\mathrm{CH}$ đến $\mathrm{CH}$ tức là khoảng cách từ các non- $\mathrm{CH}$ đến $\mathrm{CH}$ nhỏ nhất và được tính như sau:

$$
P_{d}=\sum_{i=1}^{C_{n}} \frac{1}{d_{i C H}} \rightarrow \max
$$

Trong đó: $C_{n}$ là số $n o n-C H$ trong cụm và $d_{i C H}$ là khoảng các từ nút cảm biến $i$ đến $\mathrm{CH}$. Chi tiết thuật toán FCM-CHS được trình bày như sau:

- Đầu vào: Số cảm biến $N$; Số cụm $C$; tham số mờ $m$; số vòng lặp tối đa maxSteps $>0$; và Tham số $A, B, a_{0}, d_{0}, s, k, \rho_{j}$.

- $\quad$ Đầu ra: Ma trận $U$ và Tập tâm cụm $V$.

- Chi tiết thuật toán:

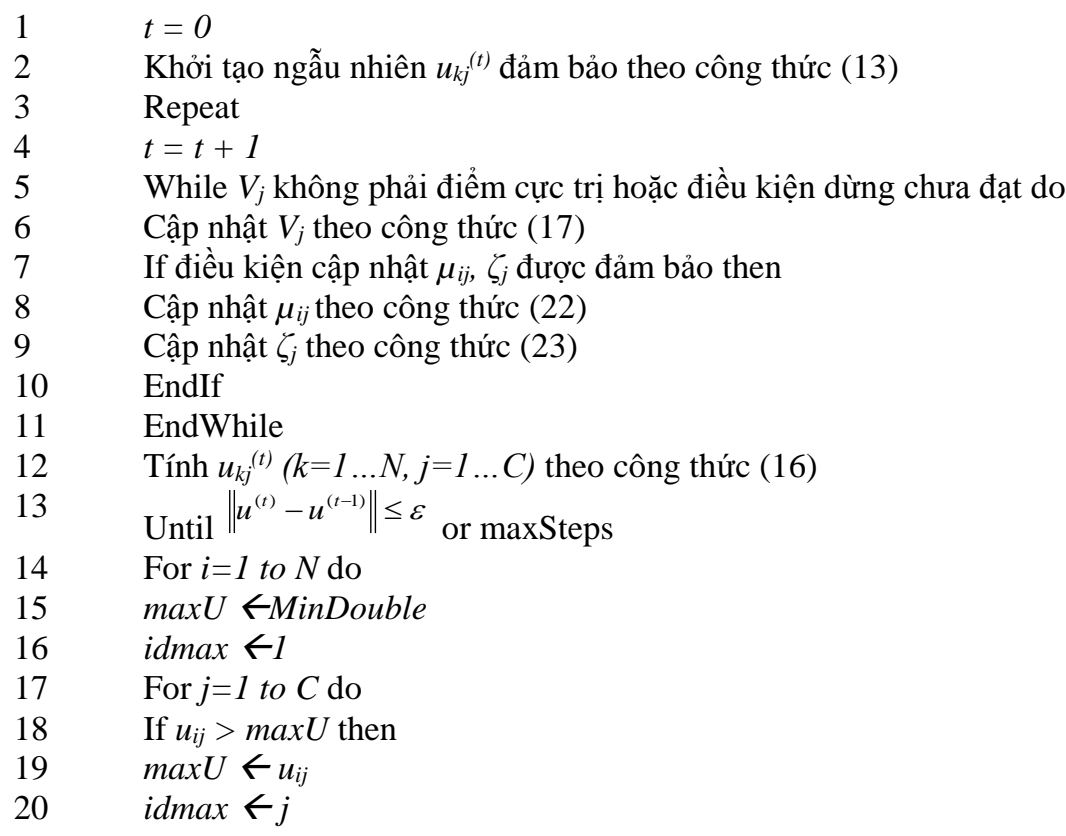




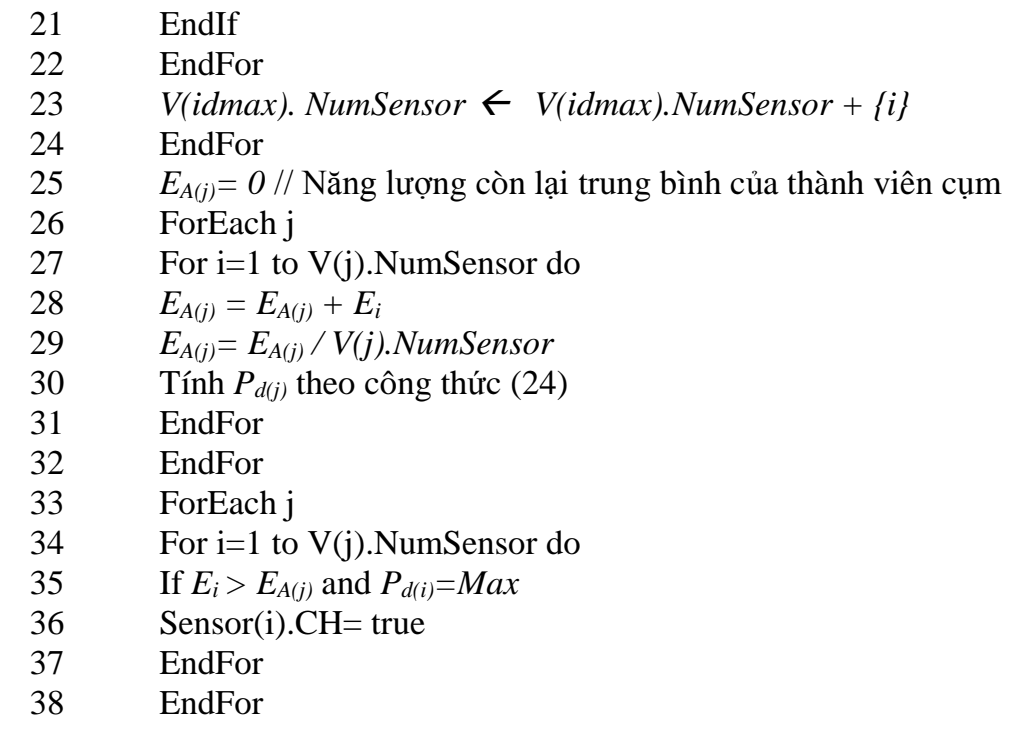

\section{THỰC NGHIẸM}

\subsection{Cài đặt}

Chúng tôi thực hiện cài đặt, so sánh kết quả các thuận toán LEACH, LEACH-C, K-Means, FCM, và FCM-CHS với các tham số được cung cấp như trong Bảng 1 . Thực nghiệm được hiện trên máy tính cấu hình CPU Intel Core i5 3.1Ghz, bộ nhớ 4GB. Các địa hình thực tế trong môi trường $3 \mathrm{D}$ được thu thập từ các vùng miền khác nhau của Việt Nam bởi phần mềm Earthexplorer (Hình 1).

\begin{tabular}{ll}
\multicolumn{2}{l}{ Bảng 1. Các tham số thuật toán } \\
\hline Tham số & Giá trị \\
\hline Năng lượng khởi tạo & $5 \mathrm{~J}$ \\
$N$ & 1000 \\
$T_{r}$ & $250 \mathrm{~m}$ \\
$E_{\text {elec }}$ & $50 \mathrm{~nJ} / \mathrm{bit}$ \\
$E_{D A}$ & $5 \mathrm{pJ} / \mathrm{bit}$ \\
$\varepsilon_{m p}$ & $0.0013 \mathrm{pJ} / \mathrm{bit} / \mathrm{m}^{4}$ \\
$\varepsilon_{f s}$ & $10 \mathrm{pJ} / \mathrm{bit} / \mathrm{m}^{2}$ \\
$l$ & $\mathrm{bytes}$ \\
\hline
\end{tabular}




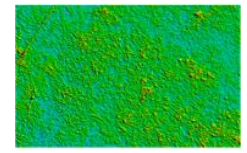

(a)

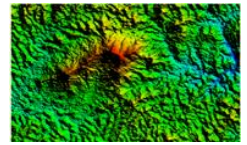

(f)

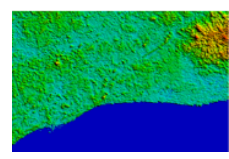

(b)

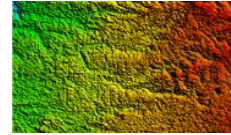

(g)

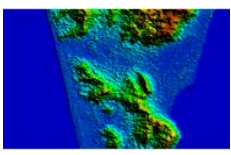

(c)

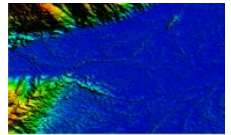

(h)

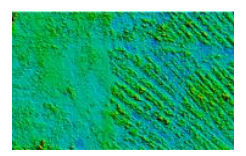

(d)

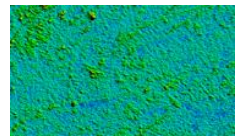

(i)

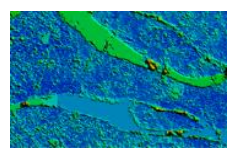

(e)

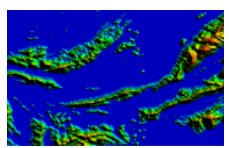

(j)

Hình 1. Các địa hình $D E M$ của Việt Nam

Ghi chú: a) Địa hình T1; b) Địa hình T2; c) Địa hình T3; d) Địa hình T4; e) Địa hình T5; f) Địa hình T6; g) Địa hình T7; h) Địa hình T8; i) Địa hình T9; và j) Địa hình T10.

\subsection{Kết quả thực nghiệm}

Chúng tôi thực nghiệm hiệu năng của thuật toán trên các địa hình khác nhau với số lượng cụm tương đương $10 \%$ số lượng các cảm biến. Bảng 2 cho thấy mức tiêu thụ năng lượng của mạng bởi các thuật toán cụ thể LEACH, LEACH-C, K-Means, FCM, và FCM-CHS. Rõ ràng là thuật toán FCM-CHS có mức tiêu thụ năng lượng trung bình nhỏ hơn các thuật toán khác.

Bảng 2. Năng lượng tiêu thụ của các thuật toán

\begin{tabular}{llllll}
\hline Địa hình & LEACH & LEACH-C & K-Means & FCM & FCM-CHS \\
\hline T1 & 182.160 & 293.957 & 138.247 & 174.789 & 146.245 \\
T2 & 195.159 & 155.514 & 129.667 & 162.054 & 161.324 \\
T3 & 208.515 & 514.775 & 154.227 & 169.368 & 159.238 \\
T4 & 164.012 & 384.664 & 116.199 & 119.982 & 110.253 \\
T5 & 209.050 & 240.831 & 187.040 & 179.560 & 120.254 \\
T6 & 173.954 & 517.287 & 144.514 & 102.553 & 85.263 \\
T7 & 212.243 & 189.875 & 167.110 & 116.225 & 96.245 \\
T8 & 240.986 & 451.496 & 175.418 & 112.452 & 99.254 \\
T9 & 191.596 & 234.403 & 134.354 & 98.457 & 92.368 \\
T10 & 152.167 & 435.096 & 92.175 & 114.452 & 108.247 \\
\hline
\end{tabular}

Hình 2 biểu thị giá trị trung bình của năng lượng tiêu thụ của tất cả các thuật toán. Chi tiết năng lượng tiêu thụ trung bình của thuật toán FCM cải tiến là 134.99. Trong khi đó, năng lượng tiêu thụ trung bình của các thuật toán khác lần lượt là 192.98 (LEACH); 341.79 (LEACH-C); 143.99 (K-Means); và 134.98 (FCM). Có thể thấy rằng thuật toán FCM-CHS (117.869) là hiệu quả nhất. 


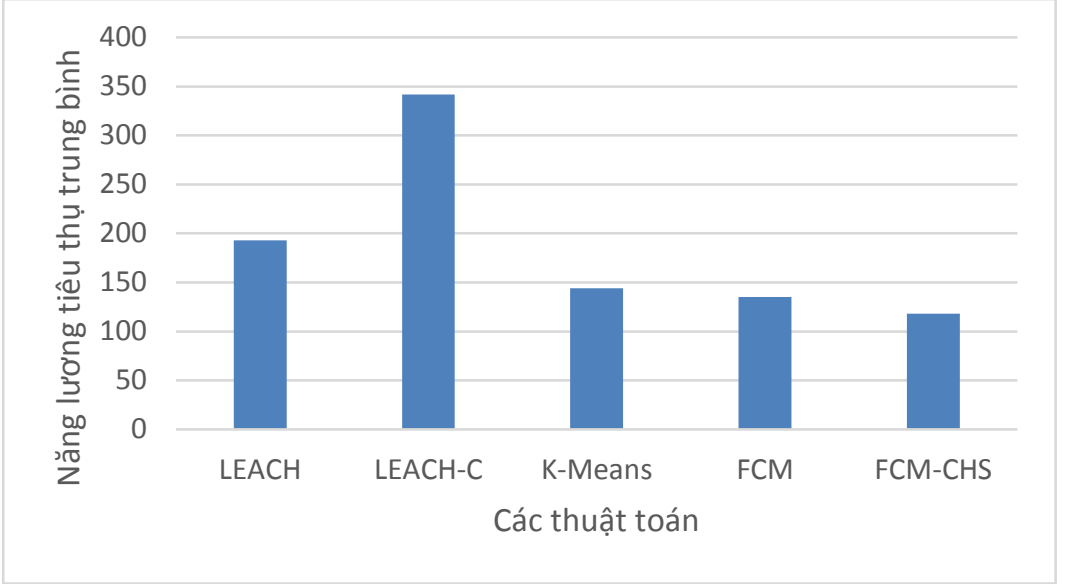

Hình 2. Năng lưọng tiêu thụ trung bình của các thuật toán

\section{KẾT LUẬn}

Trong bài báo này, chúng tôi đã nghiên cứu vấn đề tối ưu năng lượng tiêu thụ của các cảm biến, kéo dài tuổi thọ mạng bằng thuật toán phân cụm mờ kết hợp chọn nút $C H$ tối ưu. Phân cụm mờ đã chứng minh rằng hiệu quả phân cụm các thành viên có khả năng thuộc về nhiều cụm khác nhau. Tuy nhiên, phân cụm mờ truyền thống chỉ xem khoảng cách giữa các thành viên non- $\mathrm{CH}$ để hình thành cụm mà không xem xét đến khả năng kết nối giữa các $C H$ với $B S$. Thuật toán phân cụm mờ cải tiến đã khắc phục được khuyết điểm trên. Khi các cụm cảm biến được hình thành, việc lựa chọn $C H$ xoay vòng có mức năng lượng còn lại lớn và có khoảng cách gần nhất với các cảm biến trong cụm để giảm thiểu năng lượng tiêu thụ và kéo dài tuổi thọ của mạng. Qua thực nghiệm thuật toán FCM-CHS với các thuật toán phân cụm khác, kết quả nhận được là năng tiêu thụ trung bình của các cảm biến trong mạng ít nhất. Tuy nhiên, thuật toán trả giá về thời gian tính toán.

\section{TÀI LIỆU THAM KHẢO}

Akbarzadeh, V., Gagné, C., Parizeau, M., Argany, M., \& Mostafavi, M. A. (2013). Probabilistic sensing model for sensor placement optimization based on line-ofsight coverage. IEEE Transactions on Instrumentation and Measurement, 62(2), 293-303.

Bezdek, J. C., Ehrlich, R., \& Full, W. (1984). FCM: The Fuzzy C-means clustering algorithm. Computers \& Geosciences, 10(2-3), 191-203.

Hoang, D. C., Kumar, R., \& Panda, S. K. (2010). Fuzzy C-means clustering protocol for wireless sensor networks. Paper presented at The IEEE International Symposium on Industrial Electronics (ISIE), Italy.

Huang, C. F., \& Tseng, Y. C. (2005). A survey of solutions to the coverage problems in wireless sensor networks. Journal of Internet Technology, 6(1), 1-8. 
Kaur, E. S., \& Gupta, E. R. (2015). Deploying an optimized LEACH-C protocol for wireless sensor network. International Journal of Advanced Research in Computer Science and Software Engineering, 5(7), 25-30.

Mostafa, B., Abderrahmane, H., \& Saad, C. (2015). Low energy adaptive clustering hierarchy for three-dimensional wireless sensor network. Recent Advances in Communications, 15, 214-218.

Nguyen, T. T., Dang, T. H., Le, H. S., \& Le, T. V. (2015). Optimization for the sensor placement problem in $3 D$ environments. Paper presented at The IEEE International Conference on Networking, Sensing and Control (ICNSC15), Taiwan (China).

Tan, L., Gong, Y., \& Chen, G. (2008). A balanced parallel clustering protocol for wireless sensor networks using K-means techniques. Paper presented at The IEEE International Conference on Sensor Technologies and Applications, France.

Yick, J., Mukherjee, B., \& Ghosal, D. (2008). Wireless sensor network survey. Computer Networks, 52(12), 2292-2330.

Yin, Y., Shi, J., Li, Y., \& Zhang, P. (2006). Cluster head selection using analytical hierarchy process for wireless sensor networks. Paper presented at The IEEE International Symposium on Personal, Indoor and Mobile Radio Communications, Finland. 